\title{
The Coefficient of Variation Chart based on Median Run Length
}

\author{
Ming Ha Lee, K. S. Khee Yong Si, XinYing Chew, Man F. Lau, Patrick H. H. Then
}

\begin{abstract}
This study suggests a design of the coefficient of variation (CV) chart based on the median run length (MRL). The run length distribution of the $C V$ chart changes from highly skewed when the process is in-control to nearly symmetric when the process is out-of-control at the larger shifts. Therefore, the MRL can be used as an appropriate performance measure to provide a meaningful information for the performance of the $C V$ chart. The procedure for the design of the $C V$ chart based on the MRL will be provided. An industrial example is provided to illustrate the implementation of the CV chart.
\end{abstract}

Index Terms: coefficient of variation, control chart, median run length, percentiles of run length distribution, shift.

\section{INTRODUCTION}

For decades, the common performance measure of control charts has been the average value or the expected value of the run length distribution, which is termed as the average run length (ARL). However, the sole dependence on ARL as a performance measure of control charts has been criticized since the run length distribution is right-skewed. Therefore, many researchers have advocated using other performance measures for assessing the performance of control charts (see, for example [1]-[4]). One such measure is the percentage point (or percentile) of the run length distribution. The percentage points provide important and useful information such as the shape and the spread of the run length distribution, which is not provided by the ARL. Recently, the use of the percentage points of the run length distribution to access the performance of control charts was studied by many researchers. For example, [5] studied the percentage points of the run length distribution of the synthetic $\bar{X}$ chart to provide practitioners with a more complete understanding of the behavior of the synthetic $\bar{X}$ chart, instead of the sole dependence on ARL.

For the right-skewed run distribution, the average value is

Revised Manuscript Received on September 22, 2019.

Ming Ha Lee, Faculty of Engineering, Computing and Sciences, Swinburne University of Technology Sarawak Campus, Kuching, Malaysia. [Email: mhlee@swinburne.edu.my]

K. S. Khee Yong Si, Faculty of Engineering, Computing and Sciences, Swinburne University of Technology Sarawak Campus, Kuching, Malaysia. [Email: ssi@swinburne.edu.my]

XinYing Chew, School of Computer Sciences, Universiti Sains Malaysia, Pulau Pinang, Malaysia. [Email: xinying@usm.my]

Man F. Lau, School of Software and Electrical Engineering, Swinburne University of Technology, Melbourne, Australia. [Email: elau@ @swin.edu.au]

Patrick H. H. Then, Faculty of Engineering, Computing and Sciences, Swinburne University of Technology Sarawak Campus, Kuching, Malaysia. [Email: pthen@swinburne.edu.my] greater than its median. As such, the 50th percentile of the run length distribution, that is the median run length (MRL) can be used as a better performance measure to increase practitioners' confidence in understanding a control chart. Recent studies on control charts based on the MRL were made by [6]-[11].

The percentiles of the run length distribution are recommended in this study to describe the center and the spread since the shape of the run length distribution of the coefficient of variation (CV) chart changes in accordance to the magnitude of shifts, ranging from highly skewed when the shift is very small to approximately symmetric when the shift is very large. This study also suggests the design of the $\mathrm{CV}$ chart based on the MRL. The organization of this paper is as follow: The CV chart and the run length distribution of the $\mathrm{CV}$ chart are discussed in Section II. Section III provides the design of the CV chart based on the MRL. Section IV discusses the results and the finding of this study. An example of industry application is provided in Section V. Finally, conclusions are drawn in Section VI.

\section{THE COEFFICIENT OF VARIATION CHART}

For a random variable $X$, the $\mathrm{CV}$ of $X$ refers to the ratio of the standard deviation $\sigma$ to the mean $\mu$, that is $W=\sigma / \mu$. It is assumed that subgroups $\left\{X_{1 i}, X_{2 i}, X_{3 i}, \ldots, X_{n i}\right\}$ of size $n$ follows a normal distribution with mean of $\mu$ and standard deviation of $\sigma=\gamma \mu$, for sample number $i=1,2,3, \ldots$, where $\gamma$ is the population CV, then $\gamma=\sigma / \mu=a \gamma_{0}$. Here, $\gamma_{0}$ is the in-control target $\mathrm{CV}$ value and the $a$ value is the shift in the $\mathrm{CV}$. Assume that there is independence within and between these subgroups, then the sample $\mathrm{CV}$ is defined as

$$
W_{i}=\frac{S_{i}}{\bar{X}_{i}},
$$

where

$$
\bar{X}_{i}=\frac{\sum_{k=1}^{n} X_{k i}}{n}
$$

is the sample mean and

$$
S_{i}=\sqrt{\frac{\sum_{k=1}^{n}\left(X_{k i}-\bar{X}_{i}\right)^{2}}{n-1}}
$$

is the sample standard deviation, with the sample statistic $W_{i}$ is plotted on the $\mathrm{CV}$ chart.

According to [12], the cumulative distribution function (cdf) of $W$ is 


$$
F_{W}(w \mid n, \gamma)=1-F_{t}\left(\frac{\sqrt{n}}{w} \mid n-1, \frac{\sqrt{n}}{\gamma}\right),
$$

where $F_{t}\left(\cdot \mid n-1, \frac{\sqrt{n}}{\gamma}\right)$ is the cdf of the non-central $t$-distribution with $(n-1)$ degrees of freedom and non-centrality parameter $\sqrt{n} / \gamma$. Inverting the cdf in (4) gives the inverse of the cdf of $W$ as follows:

$$
F_{W}^{-1}(\alpha \mid n, \gamma)=\frac{\sqrt{n}}{F_{t}^{-1}\left(1-\alpha \mid n-1, \frac{\sqrt{n}}{\gamma}\right)},
$$

where $F_{t}^{-1}\left(\cdot \mid n-1, \frac{\sqrt{n}}{\gamma}\right)$ is the inverse of cdf of the non-central $t$-distribution with $(n-1)$ degrees of freedom and non-centrality parameter $\frac{\sqrt{n}}{\gamma}$.

The CV remains constant when the process is in-control. The process is out-of-control when the occurrence of an assignable cause due to the changes in the relation between $\mu$ and $\sigma$ such that there is a shift in the $\mathrm{CV}$. The $\mathrm{CV}$ chart was proposed by [13] for monitoring changes in the CV. Given the the significance level $p$ (i.e. type I error probability of the CV chart), the lower and upper control limits of the CV chart are given as

$$
\mathrm{LCL}=F_{W}^{-1}\left(\frac{p}{2} \mid n, \gamma_{0}\right)=\frac{\sqrt{n}}{F_{t}^{-1}\left(1-\frac{p}{2} \mid n-1, \frac{\sqrt{n}}{\gamma_{0}}\right)}
$$

and

$$
\mathrm{UCL}=F_{W}^{-1}\left(1-\frac{p}{2} \mid n, \gamma_{0}\right)=\frac{\sqrt{n}}{F_{t}^{-1}\left(\frac{p}{2} \mid n-1, \frac{\sqrt{n}}{\gamma_{0}}\right)},
$$

respectively. The performance of the $\mathrm{CV}$ chart proposed by [13] is evaluated based on the ARL. The ARL is the expected number of samples for the control chart to give an out-of-control signal at a particular shift $a$ in the $\mathrm{CV}$, where an assignable cause results in a shift of the on-target $\mathrm{CV}$ value $\gamma_{0}$ to an off-target $\mathrm{CV}$ value $\gamma_{1}=a \gamma_{0}$. Note that $a>1$ represents an increasing shift in the $\mathrm{CV}$, while $0<a<1$ represents a decreasing shift in the CV. The value of $a>1$ is considered in this study since increasing in the $\mathrm{CV}$ shift indicates the deterioration in the process. According to [14], the ARL of the CV chart is computed as

$$
\mathrm{ARL}=\frac{1}{p},
$$

where $p=1-\operatorname{Pr}(\mathrm{LCL}<W<\mathrm{UCL})$

$$
=1-\left[F_{W}(\mathrm{UCL} \mid n, \gamma)-F_{W}(\mathrm{LCL} \mid n, \gamma)\right] .
$$

Rearranging this equation gives

$$
p=F_{t}\left(\frac{\sqrt{n}}{\mathrm{UCL}} \mid n-1, \frac{\sqrt{n}}{\gamma}\right)+F_{t}\left(\frac{\sqrt{n}}{\mathrm{LCL}} \mid n-1, \frac{\sqrt{n}}{\gamma}\right)-1,
$$

where $\gamma=\gamma_{0}$ (with $a=1$ ) when the process is in-control, while $\gamma=\gamma_{1}=a \gamma_{0}$ (with $a>1$ ) when the process is out-of-control.

In this study, the run length distribution of the $\mathrm{CV}$ chart is studied to provide useful information regarding the performance of the $\mathrm{CV}$ chart as the study of the run length distribution provides a complete understanding on the behavior of the $\mathrm{CV}$ chart. The run length distribution is characterized by the percentage points or percentiles of its distribution and the percentage points are more informative than the ARL. For example, the percentage points provide information regarding the spread of the run length distribution. The lower percentage points when the process is in-control, such as the 5th and 10th percentiles provide information about the early false alarm rates.

In general, the probability mass function (pmf) of the run length distribution of a control chart is defined as

$$
\operatorname{Pr}(\mathrm{RL}=l)=p^{l-1}(1-p)
$$

and the cdf of the run length distribution can be computed as

$$
\operatorname{Pr}(\mathrm{RL} \leq l)=1-p^{l},
$$

where $l=1,2,3, \ldots$, then the $100 \tau(0<\tau<1)$ percentage point of the run length distribution can be calculated as $m_{\tau}$ such that

$$
\operatorname{Pr}\left(\mathrm{RL} \leq m_{\tau}-1\right) \leq \tau \text { and } \operatorname{Pr}\left(\mathrm{RL} \leq m_{\tau}\right)>\tau .
$$

Note that $\mathrm{ARL}=\mathrm{ARL}_{0}$ and $\mathrm{MRL}=\mathrm{MRL}_{0}$ when the process is in-control, while $A R L=A R L_{1}$ and $M R L=M R L_{1}$ when the process is out-of-control, where $\mathrm{ARL}_{0}$ and $\mathrm{MRL}_{0}$ are the in-control ARL and MRL, respectively; whereas $\mathrm{ARL}_{1}$ and $\mathrm{MRL}_{1}$ are the out-of-control ARL and MRL, respectively.

\section{THE DESIGN OF THE COEFFICIENT OF VARIATION CHART}

The design procedure of the $\mathrm{CV}$ chart is implemented as follows:

(1) Specify the values of $a, n, \mathrm{MRL}_{0}$ and $\gamma_{0}$.

(2) Solve for the $p$ value when $a=1$, to obtain the desired $\mathrm{MRL}_{0}$ value in Step (1) using (11) and (12) with $\tau=0.5$.

(3) Compute the LCL and UCL when $a=1$ based on the $p$ value in Step (2) using (6) and (7), respectively. Then, substitute the LCL and UCL values into (9) to obtain the $p$ value when $a>1$.

(4) Calculate the $\mathrm{MRL}_{1}$ using (11) and (12) with $\tau=0.5$ based on the $p$ value in Step (3) when $a>1$.

Note that in Step (1), the $a$ value is the magnitude of shift in the CV that must be identified and eliminated as soon as possible. The $\gamma_{0}$ value is usually estimated using the in-control retrospective data during Phase-I analysis. The $n$ value is determined based on the available resources depending on the number of items that can be conveniently handled in one sample [15]. 


\section{RESULT AND FINDING}

The UCL and LCL in Table I(a) are computed based on the ARL, while the UCL and LCL in Table I(b) are computed based on the MRL, for $n=5$ with $\mathrm{ARL}_{0} \in\{200,370.37,500$, $1000\}$ or $\mathrm{MRL}_{0} \in\{200,370,500,1000\}$. It can be observed that as the $\mathrm{ARL}_{0}$ or $\mathrm{MRL}_{0}$ increases, the UCL increases while the LCL decreases.

Tables II(a)-(c) provide the ARL and the run length profiles of the $\mathrm{CV}$ chart for $\gamma_{0}=0.05, n=5$ and $\mathrm{ARL}_{0}=$ 370.37. Note that $p=0.0027$ for $\mathrm{ARL}_{0}=370.37$, then the control limits of the $\mathrm{CV}$ chart are computed as $\mathrm{LCL}=$ 0.00812459 and UCL $=0.10586847$ using (6) and (7), respectively.

The lower percentiles of the in-control run length distribution in Table II(a) provide information about the early false alarm rates. For example, it can be concluded that there is a $5 \%$ chance that a false out-of-control signal will occur by the 19th sample when the process is in-control (i.e. 5 th percentile $=19$ when $a=1$ ).

The results shown in Table II(b) are expected as the ARLs are all larger than the corresponding MRLs (i.e. 50th percentiles) for all the given shifts. The run length distribution is right-skewed since its mean is larger than its median. From Table II(b), the CV chart has $\mathrm{ARL}_{0}$ of 370.37 but half of the in-control run lengths are less than 257 (i.e. $\left.\mathrm{MRL}_{0}=257\right)$. Furthermore, it is observed that the $\mathrm{ARL}_{0}$ of 370.37 falls between the 60th and 70th percentiles of the run length distribution, indicating the in-control run length distribution is skewed to the right. The results also show that the out-of-control run length distribution is also skewed to the right. For example, $\mathrm{ARL}_{1}=43.55$, whereas $\mathrm{MRL}_{1}=30$ for $a=1.25$, where the $\mathrm{ARL}_{1}$ is larger than the $\mathrm{MRL}_{1}$.

From Table II(b), ARL $=370.37,43.55,10.57,4.71$ and 2.89 for $a=1,1.25,1.5,1.75$ and 2, respectively; while MRL $=257,30,7,3$ and 2 for $a=1,1.25,1.5,1.75$ and 2, respectively. This shows that the skewness of the run length distribution decreases with the increase in the magnitude of shift $a$. In other words, the difference between the ARL and MRL decreases when the magnitude of shift $a$ increases. Due to the right-tailed run length distribution, the ARL cannot be used as the typical performance measure of the CV chart. Therefore, it is insufficient to interpret the performance of the $\mathrm{CV}$ chart solely based on the ARL as the interpretation based on ARL can be complicated and could be misleading.

Table I(a). Control limits of the CV chart when $n=5$, $\gamma_{0}=0.05$ based on ARL

\begin{tabular}{lll} 
& \multicolumn{2}{l}{$\gamma_{0}=0.05$ based on $\mathrm{ARL}$} \\
\hline $\mathbf{A R L}_{\mathbf{0}}$ & $\mathbf{U C L}$ & $\mathbf{L C L}$ \\
\hline 200 & 0.10165760 & 0.00950856 \\
370.37 & 0.10586847 & 0.00812459 \\
500 & 0.10785011 & 0.00752796 \\
1000 & 0.11227373 & 0.00631607 \\
\hline
\end{tabular}

$\gamma_{0}=0.05$ based on MRL

\begin{tabular}{lll} 
MRL $_{\mathbf{0}}$ & UCL & LCL \\
\hline 200 & 0.10419757 & 0.00865455 \\
370 & 0.10827079 & 0.00740567 \\
500 & 0.11020674 & 0.00686217 \\
1000 & 0.11453549 & 0.00575757 \\
\hline
\end{tabular}

Table II(a). ARL and run length profile (1st, 5th, 10th, 20th and 30th percentage points) of the $\mathrm{CV}$ chart for $\gamma_{0}=$ $0.05, n=5$ and $\mathrm{ARL}_{0}=370.37$

\begin{tabular}{lllllll}
\hline $\boldsymbol{a}$ & ARL & 1st & 5th & 10th & 20th & 30th \\
\hline 1 & 370.37 & 4 & 19 & 39 & 83 & 132 \\
1.25 & 43.55 & 1 & 3 & 3 & 10 & 16 \\
1.5 & 10.57 & 1 & 1 & 1 & 3 & 4 \\
1.75 & 4.71 & 1 & 1 & 1 & 1 & 2 \\
2 & 2.89 & 1 & 1 & 1 & 1 & 1 \\
\hline
\end{tabular}

Table II(b). ARL and run length profile (40th, 50th, 60th and 70th percentage points) of the CV chart for $\gamma_{0}=0.05$, $n=5$ and $\mathrm{ARL}_{0}=370.37$

\begin{tabular}{llllll}
\hline $\boldsymbol{a}$ & ARL & 40th & 50th & 60th & 70th \\
\hline 1 & 370.37 & 189 & 257 & 339 & 446 \\
1.25 & 43.55 & 22 & 30 & 40 & 52 \\
1.5 & 10.57 & 6 & 7 & 10 & 13 \\
1.75 & 4.71 & 3 & 3 & 4 & 6 \\
2 & 2.89 & 2 & 2 & 3 & 3 \\
\hline
\end{tabular}

Table II(c). ARL and run length profile (80th, 90th, 95th and 99th percentage points) of the CV chart for $\gamma_{0}=0.05$, $n=5$ and $\mathrm{ARL}_{0}=370.37$

\begin{tabular}{llllll}
\hline $\boldsymbol{a}$ & ARL & 80th & 90th & 95th & 99th \\
\hline 1 & 370.37 & 596 & 862 & 1109 & 1704 \\
1.25 & 43.55 & 70 & 100 & 129 & 199 \\
1.5 & 10.57 & 17 & 24 & 31 & 47 \\
1.75 & 4.71 & 7 & 10 & 13 & 20 \\
2 & 2.89 & 4 & 6 & 8 & 11 \\
\hline
\end{tabular}


$a=1$

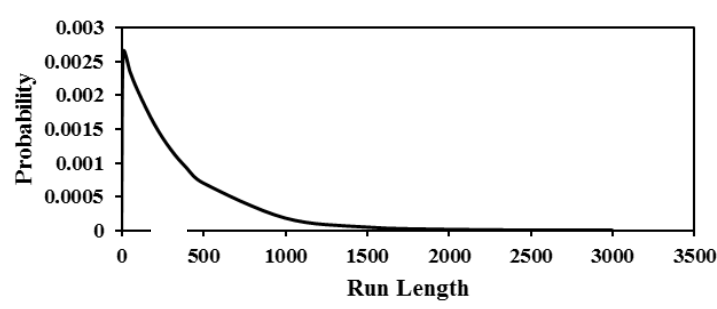

Fig. 1(a). Plot of pmf of the run length for the $\mathrm{CV}$ chart with $\gamma_{0}=0.05, n=5, \mathrm{ARL}_{0}=370.37$ and $a=1$

$$
a=\mathbf{1 . 5}
$$

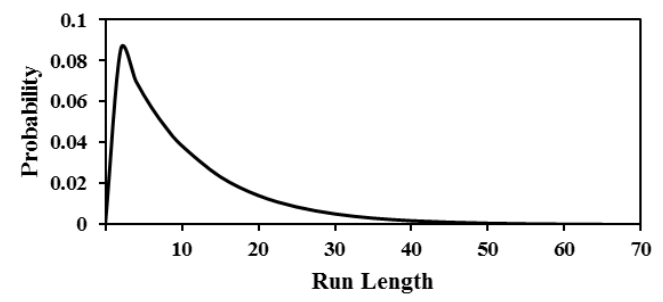

Fig. 1(b). Plot of pmf of the run length for the CV chart with $\gamma_{0}=0.05, n=5, \mathrm{ARL}_{0}=370.37$ and $a=1.5$

$$
\boldsymbol{a}=\mathbf{2}
$$

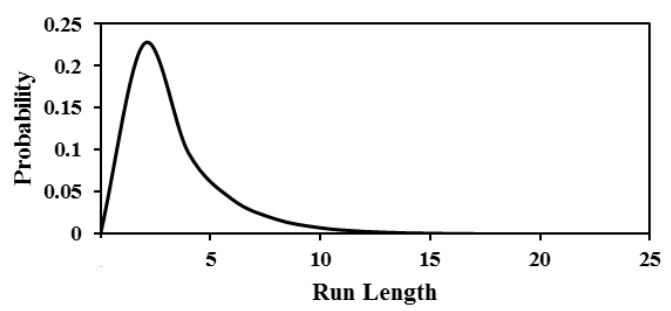

Fig. 1(c). Plot of pmf of the run length for the CV chart with $\gamma_{0}=0.05, n=5, \mathrm{ARL}_{0}=370.37$ and $a=2$

$$
a=\mathbf{3}
$$

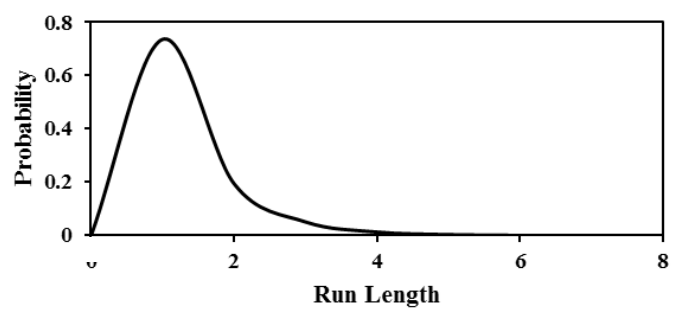

Fig. 1(d). Plot of pmf of the run length for the $\mathrm{CV}$ chart with $\gamma_{0}=0.05, n=5, \mathrm{ARL}_{0}=370.37$ and $a=3$

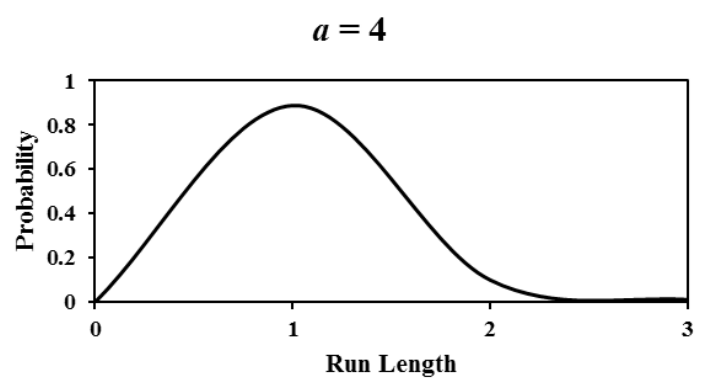

Fig. 1(e). Plot of pmf of the run length for the $\mathrm{CV}$ chart with $\gamma_{0}=0.05, n=5, \mathrm{ARL}_{0}=370.37$ and $a=4$

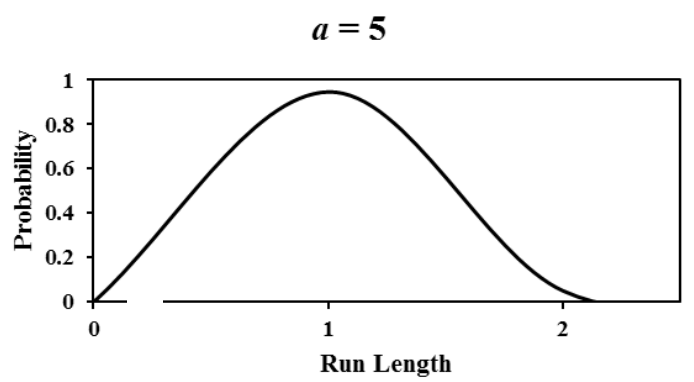

Fig. 1(f). Plot of pmf of the run length for the CV chart with $\gamma_{0}=0.05, n=5, \mathrm{ARL}_{0}=370.37$ and $a=5$

As such, the percentiles of the run length distribution can be used to provide a more meaningful interpretation. The higher percentiles of the out-of-control run length distribution in Table II(c) provide information about the out-of-control signal that can be detected at the higher probability with a certain magnitude of shift. For example, there is a probability of 0.95 that the out-of-control signal will be detected by the 129 th sample (i.e. 95 th percentile $=$ 129) when the process is shifted with a magnitude of $a=$ 1.25 .

Fig. 1(a)-(f) provide the plots of the pmf of the run length distribution for the $\mathrm{CV}$ chart with the shifts in the CV are $a=$ $1,1.5,2,3,4$ and 5, for $\gamma_{0}=0.05, n=5$ and $\mathrm{ARL}_{0}=370.37$. Fig. 1(a) shows that the run length distribution of the CV chart is highly skewed to the right when the process is in-control at $a=1$. When the process is slightly out-of-control at $a=1.5$, the run length distribution is also skewed to the right (see Fig. I(b)). However, the run length distribution is symmetric when the magnitude of shift is large at $a=5$ (see Fig. 1(f)). This shows that the skewness of the run length distribution changes according to the magnitude of shift $a$. For a highly skewed run length distribution, the interpretation based on ARL can be different compared to the symmetric run length distribution. For different skewness in the run length distribution at different magnitude of shifts, the interpretation based on ARL can be difficult. On the other hand, the interpretation based on MRL would not have the same problem, in which the interpretation based on MRL can be more meaningful and readily understood.

\section{AN EXAMPLE FOR INDUSTRIAL APPLICATION}

In this section, the application of the $\mathrm{CV}$ chart is demonstrated using an example from a solar panel manufacturing plant. The data deal with the thickness measurement for a process in manufacturing the solar wafers. The Phase-I data contain $\mathrm{m}=25$ samples, each having $\mathrm{n}=5$ measurements, in which the sample mean $\left({ }^{\bar{X}_{i}}\right)$, sample standard deviation $(\mathrm{Si})$ and sample $\mathrm{CV}(\mathrm{Wi})$ are listed in Table III.

The assumption for the process monitoring is checked by the plot of the sample CV against the sample mean in Fig. 2. Fig. 2 shows that the $\mathrm{CV}$ is constant, indicating the standard deviation of the thickness measurement is approximately proportional to the mean. 
Table III. Phase-I dataset

\begin{tabular}{|c|c|c|c|}
\hline $\begin{array}{l}\text { Sample } \\
\text { Number } i\end{array}$ & $S_{i}$ & $\bar{X}_{i}$ & $\boldsymbol{W}_{i}$ \\
\hline 1 & 7.57 & 202.74 & 0.0373 \\
\hline 2 & 11.32 & 194.56 & 0.0582 \\
\hline 3 & 7.61 & 200.43 & 0.0380 \\
\hline 4 & 10.19 & 199.16 & 0.0512 \\
\hline 5 & 8.20 & 200.61 & 0.0409 \\
\hline 6 & 15.61 & 193.48 & 0.0807 \\
\hline 7 & 6.26 & 202.68 & 0.0309 \\
\hline 8 & 10.42 & 198.57 & 0.0525 \\
\hline 9 & 17.26 & 194.79 & 0.0886 \\
\hline 10 & 7.85 & 205.99 & 0.0381 \\
\hline 11 & 11.44 & 200.08 & 0.0572 \\
\hline 12 & 10.05 & 201.32 & 0.0499 \\
\hline 13 & 9.30 & 192.33 & 0.0484 \\
\hline 14 & 10.39 & 197.23 & 0.0527 \\
\hline 15 & 17.64 & 199.27 & 0.0885 \\
\hline 16 & 6.93 & 209.99 & 0.0330 \\
\hline 17 & 9.60 & 204.28 & 0.0470 \\
\hline 18 & 7.37 & 194.64 & 0.0379 \\
\hline 19 & 10.75 & 197.58 & 0.0544 \\
\hline 20 & 10.34 & 204.02 & 0.0507 \\
\hline 21 & 11.39 & 206.16 & 0.0552 \\
\hline 22 & 6.31 & 205.68 & 0.0307 \\
\hline 23 & 14.12 & 200.39 & 0.0705 \\
\hline 24 & 6.72 & 198.00 & 0.0339 \\
\hline 25 & 4.10 & 204.85 & 0.0200 \\
\hline
\end{tabular}

This means that the constant $\mathrm{CV}$ assumption is appropriate and this justifies the use of the $\mathrm{CV}$ chart for the process monitoring. The on-target $\mathrm{CV}$ value is estimated from the Phase-I data using the root-mean-square of $W$ proposed by [16] as

$$
\gamma_{0}=\sqrt{\frac{\sum_{k=1}^{n} W_{i}^{2}}{m}}=\sqrt{\frac{0.0695}{25}}=0.05273 \approx 0.05 \text {. }
$$

It is assumed that the desired $\mathrm{MRL}_{0}$ is 370 . By setting the probability of the significance level $p$ as 0.001875 , the $\mathrm{MRL}_{0}$ of 370 is obtained based on (11) and (12) with $\tau=0.5$.
Table IV. Phase-II dataset

\begin{tabular}{|c|c|c|c|}
\hline $\begin{array}{l}\text { Sample } \\
\text { Number } i\end{array}$ & $S_{i}$ & $\bar{X}_{i}$ & $W_{i}$ \\
\hline 1 & 13.80 & 201.46 & 0.0685 \\
\hline 2 & 13.16 & 199.79 & 0.0659 \\
\hline 3 & 8.97 & 199.45 & 0.0450 \\
\hline 4 & 11.44 & 194.14 & 0.0589 \\
\hline 5 & 15.97 & 199.05 & 0.0802 \\
\hline 6 & 2.62 & 199.13 & 0.0132 \\
\hline 7 & 11.31 & 194.74 & 0.0581 \\
\hline 8 & 14.71 & 199.79 & 0.0736 \\
\hline 9 & 11.36 & 202.51 & 0.0561 \\
\hline 10 & 10.77 & 200.79 & 0.0536 \\
\hline 11 & 13.33 & 203.35 & 0.0656 \\
\hline 12 & 11.85 & 197.52 & 0.0600 \\
\hline 13 & 7.75 & 206.46 & 0.0375 \\
\hline 14 & 9.61 & 203.75 & 0.0472 \\
\hline 15 & 11.25 & 205.46 & 0.0548 \\
\hline 16 & 10.32 & 206.65 & 0.0499 \\
\hline 17 & 7.78 & 198.92 & 0.0391 \\
\hline 18 & 23.14 & 192.41 & 0.1203 \\
\hline 19 & 16.43 & 203.51 & 0.0807 \\
\hline 20 & 7.00 & 198.24 & 0.0353 \\
\hline 21 & 20.38 & 209.00 & 0.0975 \\
\hline 22 & 15.67 & 208.30 & 0.0752 \\
\hline 23 & 15.75 & 190.82 & 0.0825 \\
\hline 24 & 10.33 & 200.85 & 0.0514 \\
\hline 25 & 4.55 & 205.02 & 0.0222 \\
\hline
\end{tabular}

The control limits of the CV chart can be computed using (6) and (7) as

$$
\mathrm{LCL}=F_{W}^{-1}\left(\frac{0.001875}{2} \mid 5,0.05\right)=0.00740567
$$

and

$$
\mathrm{UCL}=F_{W}^{-1}\left(1-\frac{0.001875}{2} \mid 5,0.05\right)=0.10827079,
$$

respectively. 


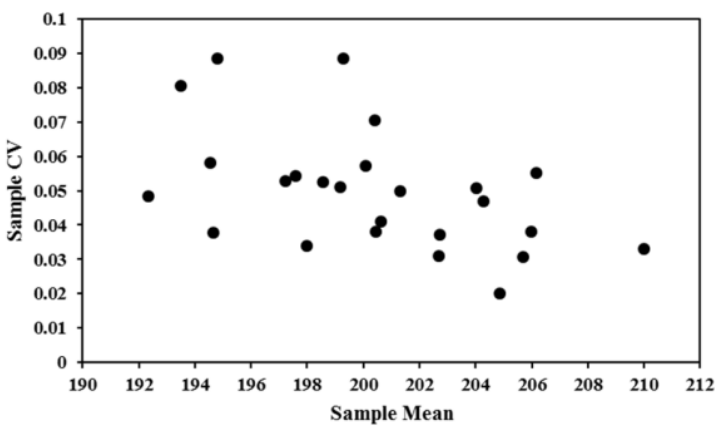

Fig. 2. Scatter plot of sample CV versus sample mean for the example

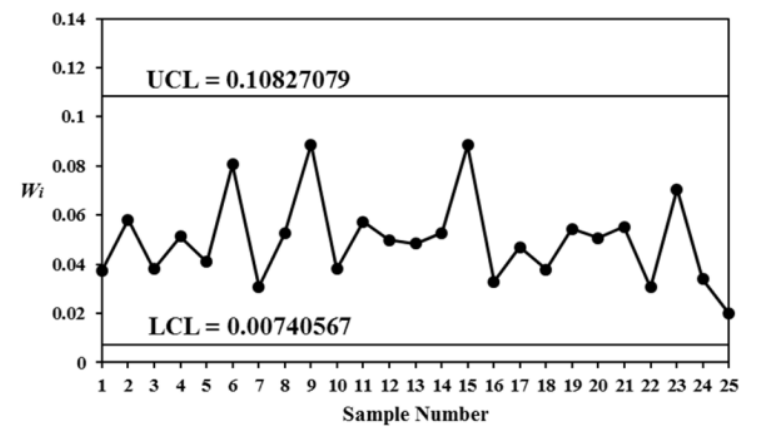

Fig. 3. CV chart for Phase-I

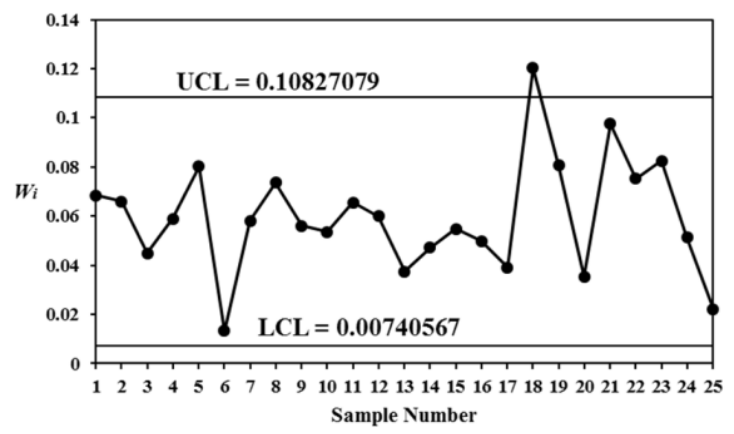

Fig. 4. CV chart for Phase-II

The CV chart for the Phase-I data is plotted in Fig. 3. Fig. 3 shows that all the samples of the Phase-I data are plotted within the control limits. This indicates that the Phase-I process is in-control. The data given in Table IV is used to construct the $\mathrm{CV}$ chart, for the Phase-II process monitoring. The increase in the $\mathrm{CV}$ is assumed to be equal to the increase in the shift size of $a=1.35$, indicating there is an increase of $35 \%$ in the $\mathrm{CV}$. In other words, the CV chart is constructed to detect a shift from $\gamma_{0}=0.05$ to $\gamma_{1}=a \gamma_{0}=1.35(0.05)=0.0675$.

As it can be noticed in Fig. 4, the out-of-control signal is detected by the CV chart at the 18th sample (see the boldfaced values in Table IV), where this sample is plotted above the UCL. Following this out-of-control signal, the underlying process is investigated by the process engineer to find the assignable cause(s), then the correction action is taken so that the process returns to the in-control state.

Using (11) and (12), the $\mathrm{MRL}_{1}$ is calculated as 19; while using (8), the $\mathrm{ARL}_{0}$ and $\mathrm{ARL}_{1}$ are computed as 533.33 and 26.95, respectively. This shows that $\mathrm{MRL}_{0}<\mathrm{ARL}_{0}$ when the process is in-control and $\mathrm{MRL}_{1}<\mathrm{ARL}_{1}$ when the process is out-of-control, indicating that the in-control and out-of-control run length distributions are both skewed to the right. This concludes that the MRL can be used as an alternative performance measure for the $\mathrm{CV}$ chart. Furthermore, since $\mathrm{MRL}_{1}=19$, the CV chart signals at or before the 19 th sample at $50 \%$ of the time.

\section{CONCLUSION}

The performance measure used for the CV chart in this study is the percentile of the run length distribution, particularly the MRL. This study shows that the shape of the run length distribution of the $\mathrm{CV}$ chart changes in accordance to the magnitude of shifts, ranging approximately symmetric when the shift is very large to highly skewed when the process is in-control. This means that the skewness of the run length distribution decreases when the magnitude of shift increases. Thus, the ARL should be not used as the sole performance measure of the CV chart. On the other hand, the MRL can be used as an alternative performance criterion, to provide a more meaningful explanation about the in-control and out-of-control performances of the $\mathrm{CV}$ chart. Moreover, a better understanding of the performance of the $\mathrm{CV}$ chart via the use of MRL helps to increase practitioners' confidence as the MRL is easier to interpret and comprehend than the ARL when the run length distribution is skewed.

\section{ACKNOWLEDGMENT}

The authors would like to acknowledge the Sarawak Multimedia Authority (SMA) in financing this research through Sarawak Digital Economy Research Grant.

\section{REFERENCES}

1. F. F. Gan, "Computing the percentage points of the run length distribution of an exponentially weighted moving average control chart," Journal of Quality Technology, vol. 23, no. 4, 1991, pp. 359-365.

2. S. Chakraborti, "Run length distribution and percentiles: The Shewhart $\bar{X}$ chart with unknown parameters," Quality Engineering, vol. 19, no. 2, 2007, pp. 119-127.

3. W. L. Teoh, M. B. C. Khoo, S. Y. Teh, "Optimal designs of the median run length based double sampling $\bar{X}$ chart for minimizing the average sample size," PLoS ONE, vol. 8, no. 7, 2013, e68580.

4. H. W. You, M. B. C. Khoo, P. Castagliola, L. Qu, "Optimal exponentially weighted moving average $\bar{X}$ charts with estimated parameters based on median run length and expected median run length," International Journal of Production Research, vol. 54, no. 17, 2016, pp. 5073-5094.

5. Z. L. Chong, M. B. C. Khoo, H. W. You, "A study on the run length distribution of synthetic $\bar{X}$ chart," International Journal of Engineering and Technology, vol. 8, no. 5, 2016, pp. 371-374.

6. W. S. Chin, M. B. C. Khoo, "A study of the median run length (MRL) performance of the EWMA $t$ chart for the mean," South African Journal of Industrial Engineering, vol. 23, no. 3, 2012, pp. 42-55.

7. M. B. C. Khoo, V. H. Wong, Z. Wu, P. Castagliola, "Optimal design of the synthetic chart for the process mean based on median run length," IIE Transactions, vol. 44, no. 9, 2012, pp. 765-779.

8. C. K. Low, M. B. C. Khoo, W. L. Teoh, Z. Wu, "The revised $m$-of- $k$ runs rule based on median run length," Communications in Statistics Simulation and Computation, vol. 41, no. 8, pp. 1463-1477, 2012.

9. M. H. Lee, M. B. C. Khoo, "Optimal designs of multivariate synthetic $|\mathrm{S}|$ control chart based on median run length," Communications in Statistics Theory and Methods, vol. 46, no. 6, 2017, pp. 3034-3053.

10. M. H. Lee, M. B. C. Khoo, "Optimal design of synthetic $n p$ control chart based on median run length," Communications in Statistics - Theory and Methods, vol. 46, no. 17, 2017, pp. 8544-8556. 
11. S. Y. Nyau, M. H. Lee, M. L. D. Wong, "Optimal statistical design of variable sample size multivariate exponentially weighted moving average control chart based on median run-length," Quality Technology \& Quantitative Management, vol. 14, no. 4, 2017, pp. 478-495.

12. B. Iglewicz, R. H. Myers, R. B. Howe, "On the percentage points of the sample coefficient of variation," Biometrika, vol. 55, no. 3, 1968, pp. 580-581.

13. C. W. Kang, M. S. Lee, Y. J. Seong, D. M. Hawkins, "A control chart for the coefficient of variation," Journal of Quality Technology, vol. 39, no. 2, 2007, pp. 151-158.

14. M. E. Calzada, S. M. Scariano, "A synthetic control chart for the coefficient of variation," Journal of Statistical Computation and Simulation, vol. 83 no. 5, 2013, pp. 853-867.

15. M. R. Reynolds Jr, J. C. Arnold, "EWMA control charts with variable sample sizes and variable sampling intervals," IIE Transactions, vol. 33, no. 6, 2001, pp. 511-530.

16. J. E. Connett, W. W. Lee, "Estimation of the coefficient of variation from laboratory analysis of split specimens for quality control in clinical trials," Controlled Clinical Trials, vol. 11, no. 1, 1990, pp. 24-36.

\section{Authors Profile}

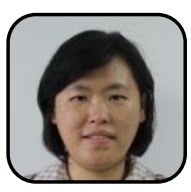

Ming Ha Lee is a senior lecturer in the Faculty of Engineering, Computing and Science at Swinburne University of Technology Sarawak Campus, Malaysia. She received her BTech from Universiti Sains Malaysia, her MSc from Universiti Putra Malaysia and her PhD from Universiti Sains Malaysia.

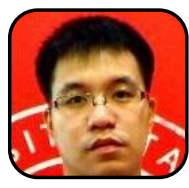

K. S. Khee Yong $\mathbf{S i}$ is a full time master student by research mode. He currently studies in the Faculty of Engineering, Computing and Science, Swinburne University of Technology Sarawak Campus. He obtained his Bachelor of Engineering in Electrical and Electronic Campus. from Swinburne University of Technology Sarawak

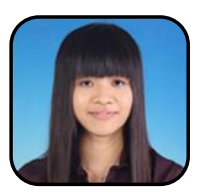

XinYing Chew is a senior lecturer in the School of Computer Sciences, Universiti Sains Malaysia. She holds a $\mathrm{PhD}$ in statistical quality control from University Sains Malaysia. She is a certified trainer with Human Resources Development Fund, Malaysia. Her research papers are published in several renowned international peer-reviewed journals, such as Computers \& Industrial Engineering, Quality and Reliability Engineering International and etc. She is also an adjunct research fellow of Swinburne University of Technology Sarawak Campus. Prior to her academic career, she worked in the Advanced Analytics research team of a renown U.S. multinational company. Her areas of research are in advanced analytics and statistical quality / process control.

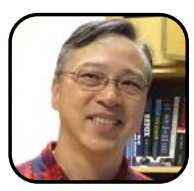

Man F. Lau received his BSc (Hons) from The University of Hong Kong and $\mathrm{PhD}$ from The University of Melbourne. $\mathrm{He}$ joined Swinburne University of Technology in 2000 He received 2 ARC DP research grants in 2005 (a 3-year ARC DP project) and 2007 (a 5-year ARC DP project). His currently research interests are on Data Mining and Testing Prediction Models. Currently, he is working on a Big Data project on testing Optimal Oil Drilling Prediction Models based on past 15+ years of oil drilling data.

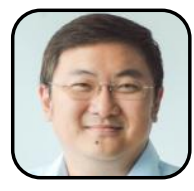

Patrick H. H. Then is the director for the Centre for Digital Futures Swinburne Sarawak. $\mathrm{He}$ is a strong advocate of R\&D and commercialization of innovations in Big Data, Data Mining and Internet of Things. He has established industry collaboration at national and international levels. He has been leading multiple industry-funded projects in research and development in collaboration with prominent ICT partners such as Sarawak Information Systems Sdn Bhd (SAINS), IDS (Malaysia) Sdn Bhd in Sarawak, and organizations around the world. He has established partnership between Swinburne and international commercial partners such as Fusionex International Ltd, UK, D\&J Human Care, South Korea, and Easy Global Market, France. He is a member of the Institute of Electrical and Electronics Engineers USA and the Australian Computer Society. 